\title{
Primary Cicatricial Alopecia - Clinicopathological Study from South Kerala
}

\author{
Sruthy Manikkath ${ }^{1}$, Beena Sunny², Rani Mathew ${ }^{3}$ \\ 1,2 Department of Dermatology \& Venereology, Government T D Medical college, Alappuzha, Kerala, India. \\ ${ }^{3}$ Department of Dermatology \& Venereology, Government Medical College, Thiruvananthapuram, Kerala, India.
}

\section{ABSTRACT}

\section{BACKGROUND}

Cicatricial alopecia is the term used for permanent areas of hair loss due to destruction of hair follicles. It may occur due to a disease that primarily affects hair follicles or due to external causes that damages hair follicle secondarily. The common causes of primary cicatricial alopecia include discoid lupus erythematosus, lichen plano pilaris, pseudopelade of Brocq, folliculitis decalvans and folliculitis keloidalis. Exact diagnosis at an early stage will help to provide disease specific management at the earliest thus, preventing further progression of the disease. We conducted this study to assess the role of clinico-pathological correlation to find out the exact aetiology of primary scarring alopecia in our patients.

\section{METHODS}

We conducted a descriptive cross sectional study to find out aetiology of primary cicatricial alopecia based on clinical and histopathological findings of 50 patients who attended the outpatient department of Dermatology in our hospital. After a detailed history and clinical examination of scalp, biopsy of lesional scalp skin was taken in all patients. In relevant cases direct immunofluorescence study was also done.

\section{RESULTS}

Age of the patients ranged from 16-75 years. M: F ratio was 1:1.5. Hair loss, itching, scaling and scalp pain were the reported symptoms. Commonly affected sites were parietal and temporal areas. $76 \%$ of patients had multiple lesions. Absent follicular ostia, follicular plugging, atrophy, pigmentary changes, scaling and telangiectasias were the commonly observed clinical scalp findings. $88 \%$ of cases showed lymphocytic infiltrate on histopathology. After clinico-pathological correlation, 48\% patients had discoid lupus erythematosus followed by follicular lichen planus in $28 \%$ and pseudopelade of Brocq in $20 \%$ cases. Folliculitis decalvans and folliculitis keloidalis each were observed in $2 \%$ cases of primary scarring alopecia.

\section{CONCLUSIONS}

Clinicopathological correlation is highly useful to make an early diagnosis in primary cicatricial alopecia. In our patients discoid lupus erythematosus was found to be the predominant cause.

\section{KEY WORDS}

Cicatricial Alopecia, Clinicopathological Correlation, Discoid Lupus Erythematosus, Lichen Planus
Corresponding Author: Dr. Rani Mathew,

Associate Professor, Dept. of Dermatology\& Venereology, Government Medical College, Thiruvananthapuram, Kerala, India. E-mail: raniguild@yahoo.co.in

DOI: $10.14260 /$ jemds/2021/809

How to Cite This Article: Manikkath S, Sunny B, Mathew R. Primary cicatricial alopecia- clinicopathological study from South Kerala. J Evolution Med Dent Sci 2021;10(45):4009-4012, DOI: 10.14260/jemds/2021/809

Submission 20-11-2021, Peer Review 28-11-2021, Acceptance 20-12-2021, Published 31-12-2021.

Copyright (C) 2021 Sruthy Manikkath et al. This is an open access article distributed under Creative Commons Attribution License [Attribution 4.0 International (CC BY 4.0)] 


\section{BACKGROUND}

Primary cicatricial alopecia (PCA) is a group of disorders where there is inflammation and destruction of hair follicles finally ending in irreversible hair loss. ${ }^{1}$ Secondary scarring alopecia results from disease process external to hair follicles and include traumatic alopecia, sclerosing disorders, granulomatous conditions like sarcoidosis, bacterial, viral \& mycobacterial infections and neoplastic conditions. ${ }^{2}$ In a workshop sponsored by North American Hair research society (NAHRS), a classification of PCA based on predominant inflammatory infiltrate was developed. It includes 1) Lymphocytic- Chronic cutaneous lupus erythematosus, lichen planus pigmentosus (LPP), pseudopelade of Brocq, keratosis follicularis spinulosa decalvans, alopecia mucinosa 2) Neutrophilic- Folliculitis decalvans, dissecting cellulitis 3) Mixed- Folliculitis (acne) keloidalis 4) Nonspecific.1,2,3 Early diagnosis and treatment is essential as scarring alopecia affects the self-esteem of the patient.

No single sign is pathognomonic for a particular disease. ${ }^{2}$ So clinical history, detailed examination of scalp and scalp biopsy is needed in case of cicatricial alopecia to find out the underlying causes which will help select appropriate treatment. Localization of symptoms when present can direct the clinician to the areas of active disease. Pattern of alopecia, follicular and interfollicular stigmata, presence of other skin or mucosal lesions and systemic symptoms will give clues to diagnosis. Direct immunofluorescence studies of lesional skin will add to our diagnosis.

There is scarcity of research on primary cicatricial alopecia in our area. Hence this study.

\section{Aims and Objectives}

We intend to find out the aetiology of primary cicatricial alopecia based on clinical and histopathological findings in our area.

\section{METHODS}

Ours was a descriptive cross sectional study conducted in the Department of Dermatology and Venereology, in a major tertiary care centre in South Kerala.

\section{Inclusion Criteria}

All cases of primary cicatricial alopecia attending Dermatology outpatient department from Dec 2013 to May 2015.

\section{Exclusion Criteria}

1. Secondary cicatricial alopecia

2. Lesions present at birth

3. Patients unwilling for consent

After obtaining a written informed consent, a detailed history was taken regarding the symptoms, onset and progress of the disease. Complete examination of scalp was done for clinical changes. Permanent areas of hair loss with destruction of hair follicles were taken as cicatricial alopecia. We also examined for any skin, mucosal and nail lesions. $5 \mathrm{~mm}$ punch biopsy was taken from lesional scalp skin under aseptic precautions and sent for histopathology in all patients. In relevant cases scalp biopsy was sent for direct immunofluorescence also. All details were recorded in the proforma.

\section{Statistical Analysis}

SPSS statistical software was used for analysis. Qualitative data was analysed using percentages while quantitative data was analysed using mean and median.

Prior to study, permission was obtained from human ethical committee and institutional research committee of our hospital.

\section{RESULTS}

During the study period of 18 months, 50 patients presented with primary cicatricial alopecia. Age of the patients ranged from 16-75 years. Maximum number of patients (50\%) presented in $5^{\text {th }}$ and $6^{\text {th }}$ decade of life. It included thirty females and rest were males, M:F ratio being 1:1.5.Maximun number of patients reported to the OP within twelve months of onset of disease (48\%). Another $30 \%$ cases reported in 12-24 months. Among 6 patients duration of disease was more than 5 years. Hair loss was the predominant symptom reported by all patients. Itching, scaling and pain were other symptoms, seen in 26(52\%), 19(38\%) and 2(4\%) patients respectively. Most commonly affected sites were parietal and temporal area (74\% each) of scalp. Occipital area was affected in $40 \%$ cases while least affected was frontal area (20\%). Multiple well defined lesions were observed in $76 \%$, diffuse lesions in $16 \%$ and single lesion in $8 \%$ patients. Various scalp changes observed in our study are shown in Table-1.

\begin{tabular}{|ccc|}
\hline Scalp Changes & Frequency & Percentage \\
\hline Absent follicular ostia & 50 & 100 \\
Follicular plugging & 29 & 58 \\
Footprint in snow & 6 & 12 \\
Atrophy & 30 & 60 \\
Depigmentation & 17 & 34 \\
Hyperpigmentation & 25 & 50 \\
Scaling & 31 & 62 \\
Hair pull test positive & 12 & 24 \\
Erythema & 8 & 16 \\
Doll's hair & 1 & 2 \\
Telangiectasia & 13 & 26 \\
\hline Table 1. Scalp Changes Seen Clinically in Our Patients \\
\hline
\end{tabular}

Histopathological subtypes based on NAHRS working classification are shown in Table-2.

\begin{tabular}{|ccc|}
\hline Infiltrate & Frequency & Percentage \\
Lymphocytic & 44 & 88 \\
Neutrophilic & 1 & 2 \\
Mixed & 1 & 2 \\
Nonspecific & 4 & 8 \\
\hline Table 2. Distribution According to Type of Infiltrates \\
\hline
\end{tabular}

Table -3 shows the various histopathological features in fifty patients. 


\begin{tabular}{|cccccc|}
\hline Histopathology & DLE & LPP & PPB & FD & FK \\
Hyperkeratosis & + & - & - & - & + \\
Epidermal atrophy & + & - & - & - & - \\
Follicular plugging & + & $+/-$ & - & - & + \\
Wedge shaped hyper granulosis & - & + & - & - & - \\
Basal cell damage & + & + & - & - & - \\
Basement membrane thickening & + & - & - & - & - \\
Lichenoid infiltrate & - & + & - & - & - \\
Pigment incontinence & + & + & - & - & - \\
Lymphocytic infiltrate & + & + & + & - & - \\
Neutrophilic infiltrate & - & - & - & + & - \\
Mixed infiltrate & - & - & - & - & + \\
Fibrous tracts & + & $+/-$ & + & + & + \\
Mucin & + & - & - & - & - \\
\hline Table 3. Histopathological Features & & \\
\hline \multicolumn{6}{|r}{}
\end{tabular}

DLE- discoid lupus erythematosus, LPP-lichen plano pilaris, PPB- pseudopelade of Brocq, FD- folliculitis decalvans, FK- folliculitis keloidalis

After clinico-pathological correlation, we made our final diagnosis. In the present study $48 \%$ patients had discoid lupus erythematosus (DLE), $28 \%$ had lichen planus pigmentosus (LPP), $20 \%$ pseudopelade of Brocq and $2 \%$ each had folliculitis decalvans and folliculitis keloidalis. Details are summarized in Table- 4 .

\begin{tabular}{|cccc|}
\hline Diagnosis & Clinical & Pathological & Final \\
Discoid lupus erythematosus & 20 & 24 & 24 \\
Lichen plano pilaris & 14 & 14 & 14 \\
Pseudopelade of Brocq & 6 & 6 & 10 \\
Folliculitis decalvans & 1 & 1 & 1 \\
Folliculitis keloidalis & 1 & 1 & 1 \\
Nonspecific & 8 & 4 & 0 \\
\hline Table 4. Diagnosis- Clinical, Pathological and Final \\
\hline
\end{tabular}

\section{Primary Cicatricial Alopecia Due to DLE}

Among 24 patients with DLE 8 (33.3 \%) were males and rest (66.6\%) were females. Most affected age group was 40-60 years. Mean age was 40 yrs and median distribution of duration was 12 months. Temporal and parietal area was maximally involved (79.2 \% each). Multiple lesions were seen in $75 \%$ and single patch in $4.2 \%$ patients. Diffuse lesions were observed in $20.8 \%$ cases. Presenting complaints were hair loss, scaling, pruritus and scalp pain in $100 \%, 60 \%, 42$ $\%$, and $4 \%$ of patients respectively.

Characteristic scalp changes observed clinically were follicular plugging in 16 patients, epidermal atrophy in 22, telangiectasias in 13, depigmentation in 15 and hyperpigmentation in 11 cases.

Ten patients had DLE lesions elsewhere on skin, while 8 had mucosal lesions. DIF was done in 11 patients with doubtful diagnosis. Six cases (54.5\%) showed positive lupus band (IgG \& C3 in 3, IgG in 2 and IgM in 1 case).

\section{Lichen Planus of Scalp}

Fourteen patients had PCA due to lichen planus. Mean age was 43 years. Six patients were males and rest were females. Mean duration was 2 years. 13 patients had lichen plano pilaris while one presented as frontal fibrosing alopecia. Most common sites affected were parietal and temporal (64.3\% each). Multiple well defined plaques were seen in 11 cases. Two patients had only a single plaque while in one patient the involvement was diffuse. Important scalp changes noted in our study were follicular plugging in 10 patients, atrophy in 8 , hyperpigmentation in 6 and scaling in 11 patients. All patients with clinical diagnosis of lichen planus, showed histopathological features of the same.
Of the 14 patients, 5 had skin lesions of lichen planus, 6 had mucosal lesions and 2 had nail changes.

\section{Pseudopelade of Brocq}

There were ten patients with pseudopelade, 5 males and rest were females. Mean age was 36 years and mean duration of symptoms was 1 year. Clinically classical footprint in snow appearance was seen in 6 cases. Histopathology showed epidermal atrophy and fibrous tracts replacing hair follicles. Six patients showed perifollicular lymphocytic infiltrate.

\section{Folliculitis Decalvans}

We had 1 patient with folliculitis decalvans who was a 56 year old male. Patient presented with 10 months history of itching and hair loss in tempero-parietal region. Clinically follicular pustules with tufted folliculitis (doll's hair) in the advancing margin of alopecic plaque were seen. Histopathology showed a neutrophilic infiltrate.

\section{Folliculitis Keloidalis}

37 year old male reported with itchy papules and patchy hair loss in occipital area of 15 months duration, histology showing hyperkeratosis, follicular plugging and mixed perifollicular inflammatory infiltrate of lymphocytes and plasma cells.

\section{DISCUSSION}

Most of our patients belonged to age group 40-60 years and $\mathrm{M}$ : F ratio was 1: 1.5. In one large retrospective study on cicatricial alopecia by Whiting, majority of affected adults were females (M: $\mathrm{F}=1: 2.6)$.

Most of our patients presented in first 2 years of onset of disease (78\%). Duration of disease could not be assessed correctly due to lack of visibility of lesion to the patient. Clinically all patients presented with hair loss. Absent follicular ostia was noted in all patients which is pathognomonic of cicatricial alopecia. ${ }^{2}$

Majority of our cases (88\%) had lymphocytic dermal infiltrate on histopathology. Sowjanya et al. in their study on acquired primary cicatricial alopecia, observed lymphocytic versus neutrophilic infiltrate ratio to be 7.3:1.5

We observed DLE in 48\% cases followed by LPP in $28 \%$, and pseudopelade of Brocq in $20 \%$ patients. Folliculitis decalvans and folliculitis keloidalis were seen in $2 \%$ patients each. This is in contrast to another Indian study where $50 \%$ of patients had LPP, $20 \%$ had chronic cutaneous lupus erythematosus, and $12 \%$ had pseudopelade of Brocq. $6 \%$ each had keratosis follicularis spinulosa decalvans, folliculitis decalvans and acne keloidalis nuchae. ${ }^{5}$ Tan et al. reported DLE as the foremost cause (33.9 \%) followed by pseudopelade $(24.1 \%)$ and lichen plano pilaris $(22.3 \%) .{ }^{6}$ In another study by Whiting, pseudopelade of Brocq was the most common cause $(40.6 \%)$ followed by lichen plano pilaris in $12.6 \%$ and folliculitis decalvans in $11.2 \%$ patients. ${ }^{4}$ This disparity in different studies may be due to racial differences. 


\section{DLE}

24 patients had PCA due to DLE with a mean age of 40 years and M: F ratio of 1:2.In another Indian study mean age was 35.6years and M: F ratio 1:4. ${ }^{5}$ Hair loss and pruritus were the most common symptoms reported by our patients which is similar to that reported by Tan et al. but scalp pain was seen only in $4 \%$ of our patients which is in contrast with the findings of other study where $26.2 \%$ had it. ${ }^{6}$ Temporal and parietal areas were the most commonly affected areas in our study. This is in concordance with study by Harries MJ et al. where lesions were predominantly found in central distribution of scalp. ${ }^{7}$ However Tan et al. observed no such predilection. ${ }^{6}$ Scientific explanation for any site preference is lacking in literature. Main scalp changes observed were well defined alopecic plaque with absent follicular ostia, carpet tack sign, atrophy, depigmentation, hyperpigmentation and telangiectasia which is similar to that described in literature. ${ }^{2}$ Classical histopathological changes of DLE described in various studies were observed in all our clinically diagnosed cases. ${ }^{5,6,7}$ Direct immunofluorescence was done in 11 patients out of whom 6 were lupus band positive (54.5\%). Tan et al. reported positive DIF in $62.5 \%$ patients. ${ }^{6}$ According to textbooks, DIF positivity in DLE lesional skin is around $80 \%{ }^{2}$ Low DIF positivity in our study could be due to improper biopsy site or due to prior application of topical steroids. After histopathology and DIF we could classify four clinically nonspecific cases as DLE.

\section{Lichen Planus of Scalp}

Fourteen patients presented clinically to us with a mean age of 43 years and F: $M$ ratio of 1.33:1.This is in concordance with studies by Tan et al. and Sowjanya et al.5,6 $78.6 \%$ patients had multiple lesions distributed mainly in temporal and parietal regions. In another similar Indian study most of patients had multifocal lesions but there was no site of predilection. ${ }^{5}$ Clinical and histopathological features observed by us were in accordance with other studies. ${ }^{2,5,7}$ Clinico-pathological correlation was obtained in all cases.

\section{Pseudopelade of Brocq}

We had 10 patients with Pseudopelade of Brocq of whom 6 were diagnosed clinically and rest after histopathology. Mean age was 36 years with $\mathrm{M}$ : $\mathrm{F}$ ratio of $1: 1$. Slight female predominance is seen in some other studies. ${ }^{5,6}$ This difference may be due to the small number of patients with PPB in our study. Most of them presented with asymptomatic patchy hair loss with surface showing footprint in snow appearance as described in other studies. ${ }^{5,6}$ There was no evidence of DLE or lichen planus elsewhere on skin or mucosa. Histopathology of these cases showed epidermal atrophy, dermal lymphocytic infiltrates and fibrous tracts replacing follicles as observed in literature.5,6,7 In 4 patients inflammatory changes were minimal or absent on histology. It is well described that inflammation may disappear in later stages of the disease. ${ }^{8}$

\section{Folliculitis Keloidalis}

Even though folliculitis keloidalis in not an uncommon disorder, we had only 1 patient who was a 37 year old male.
Clinical and histopathological features were in concordance with those described in literature. ${ }^{5}$

\section{Folliculitis Decalvans}

Clinically we diagnosed folliculitis decalvans in a 56 year old male patient which was confirmed histopathologically.

\section{CONCLUSIONS}

In the present study we could classify all cases of primary cicatricial alopecia into specific entities with the help of clinical, histopathological and immunofluorescence findings. We could also study the clinical presentations and histopathology of each of these conditions. Improper biopsy site could be a reason for negative DIF in some cases.

To arrive at a diagnosis in primary cicatricial alopecia, clinico-pathological correlation is highly useful. Direct immunofluorescence studies makes diagnosis more accurate. Early diagnosis and treatment will help in better prognosis of scarring alopecia. We could not do immunofluorescence in all our patients. More studies with higher sample size, with immunofluorescence being done for all cases, will help us to have a more clear picture of the aetiology of primary scarring alopecia.

Data sharing statement provided by the authors is available with the full text of this article at jemds.com.

Financial or other competing interests: None.

Disclosure forms provided by the authors are available with the full text of this article at jemds.com.

\section{REFERENCES}

[1] Al-Hilo MM, Al-Saedy SJ, Yacoob PY. Primary cicatricial alopecia; a clinical and histopathological descriptive study. Am J Dermatol Venereol 2013;2(3):15-22.

[2] Messenger AG, Sinclair RD, Farrant P, et al. Acquired disorders of hair. In: Griffiths CEM, ed. Rook's textbook of dermatology. 9th edn. UK: Wiley Blackwell 2016:1395403.

[3] Ross EK, Tan E, Shapiro J. Update on primary cicatricial alopecias. J Am Acad Dermatol 2005;53(1):1-37.

[4] Whiting DA. Cicatricial alopecia: clinicopathological findings and treatment. Clin Dermatol 2001;19(2):21125.

[5] Sowjanya CL, Rao TN, Guruprasad P, et al. Clinicopathological study of acquired primary cicatricial alopecia. JNTR Univ Health Sci 2012;1:21-6.

[6] Tan E, Martinka M, Ball N, et al. Primary cicatricial alopecias: clinicopathology of 112 cases. J Am Acad Dermatol 2004;50(1):25-32.

[7] Harries MJ, Trueb RM, Tosti A, et al. How not to get scar 囚ed: pointers to the correct diagnosis in patients with suspected primary cicatricial alopecia. Br J Dermatol 2009;160(3):482-501.

[8] Otberg N, Shapiro J. Hair growth disorders. In: Goldsmith LA, ed. Fitzpatrick's dermatology in general medicine. $8^{\text {th }}$ edn. McGrow Hill Companies 2012:979-1008. 